\title{
Transitions between complex configurations in the excitation of the Double Giant Resonance
}

\author{
V.Yu. Ponomarev ${ }^{1,2 *}$ and C.A. Bertulani ${ }^{1 \dagger}$ \\ ${ }^{1}$ Instituto de Fúsica, Universidade Federal do Rio de Janeiro, 21945-970 Rio de Janeiro, RJ, Brazil \\ ${ }^{2}$ Bogoliubov Laboratory of Theoretical Physics, Joint Institute for Nuclear Research, 141980, Dubna, Russia \\ (July 13, 2021) \\ The transitions between complex configurations, to which the giant dipole resonance (GDR) \\ and the double giant dipole resonance (DGDR) doorway states are coupled, are taken into account \\ in second order perturbation theory for the reaction amplitude. It is proved that only transitions \\ between GDR and DGDR doorway states play an essential role in the Coulomb excitation of the \\ DGDR.
}

PACS numbers: 23.20.-g, 24.30.Cz, 25.70.De, 25.75.+r

*e-mail: vlad@thsun1.jinr.ru

${ }^{\dagger}$ e-mail: bertu@if.ufrj.br 
After the first observation of the double giant dipole resonance (DGDR) in relativistic heavy ion collisions (RHIC) [1.22 the magnitude of its excitation cross section attracts a special attention. This is because the first data 22 indicated a strong enhancement of the DGDR excitation in ${ }^{136} \mathrm{Xe}$ (by a factor of 2-4) as compared to the theoretical predictions available [3, 4 . Several attempts have been made to understand the reason of this phenomena 59 9]. A few years later a similar experiment with the excitation of the DGDR in ${ }^{208} \mathrm{~Pb}$ brought another intriguing news [10] which were interpreted as a good agreement between experiment and theory if some corrections to the pure harmonic picture of the DGDR excitation were added [6 9]. Unless a good systematic study is achieved, the question on the excitation cross section of the DGDR in RHIC remains open. This stimulates theoretical studies on the different processes which might be responsible for an enhancement of the DGDR strength function.

Recently it was argued that transitions between complex configurations, to which the GDR and DGDR doorway states are coupled, may be responsible for the enhanced DGDR decay into the GDR states as compared to the GDR decay into the ground state 11. However, it should be remembered that because of the available phase space two consequent $\gamma$-emissions from the DGDR is not the same as the inverse process of the DGDR excitation. The transitions between the GDR and the DGDR complex configurations were not taken into account in previous microscopic studies [9, 12, 13]. The role of these transitions for the DGDR excitation in RHIC will be considered in the present paper. It will be concluded that their role is marginal in the process under consideration although a huge amount of the E1-strength is hidden in the GDR $\rightarrow$ DGDR transition. This negative result ensures that calculations, in which only transitions between collective components of the GDR and DGDR are taken into account and which are much easier to carry out, require no further corrections.

The main mechanism for the DGDR excitation in RHIC within a semiclassical approach is a two-step process g.s. $\rightarrow$ GDR $\rightarrow$ DGDR [14. Corrections to the secondorder perturbation theory arising from coupled-channels were studied in Ref. [5]. Although for grazing impact parameters the coupled-channels calculation deviates on the $20 \%$ level from second-order perturbation theory, it makes only a small change to the total cross sections. That is, for not too small impact parameters the secondorder perturbation theory works quite well. Indeed, this has been observed recently in the analysis of the experiment on DGDR excitation in lead projectiles impinging on different $Z$-targets 10 .

Thus, we can use for the excitation probability of the DGDR

$$
P_{D G D R}\left(E_{f}, b\right)=\frac{1}{4} \sum_{M_{f}} \mid \sum_{i, M_{i}} a_{0(0) \rightarrow 1_{i}^{-}\left(M_{i}\right)}^{E 1(\mu)}\left(E_{i}, b\right)
$$

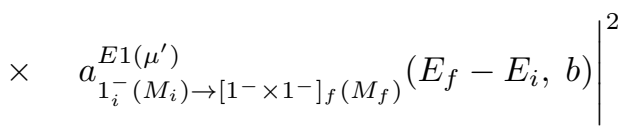

where the index $i$ labels intermediate states belonging to the GDR, and $a_{J_{1}\left(M_{1}\right) \rightarrow J_{2}\left(M_{2}\right)}^{E 1(\mu)}$ is the first-order E1 excitation amplitude for the transition $J_{1}\left(M_{1}\right) \longrightarrow J_{2}\left(M_{2}\right)$ in a collision with impact parameter $b$. For each state, $J$ and $M$ denote the total angular momentum and the magnetic projection, respectively.

The amplitude $a_{J_{1}\left(M_{1}\right) \rightarrow J_{2}\left(M_{2}\right)}^{E 1(\mu)}$ is given by

$$
\begin{aligned}
a_{J_{1}\left(M_{1}\right) \rightarrow J_{2}\left(M_{2}\right)}^{E 1(\mu)}(E, b) & =\left(J_{1} M_{1} 1 \mu \mid J_{2} M_{2}\right) \\
\times & <J_{2}\|E 1\| J_{1}>f_{E 1(\mu)}(E, b) .
\end{aligned}
$$

It is a product of the reduced matrix element < $J_{2}\|E 1\| J_{1}>$ for the $E 1$-transition between the states $J_{1}\left(M_{1}\right)$ and $J_{2}\left(M_{2}\right)$ which carries nuclear structure information and the reaction function $f_{E 1(\mu)}(E, b)$. The latter depends on the excitation energy, charge of the target, beam energy, and is calculated according to Ref. 115]. Except for the dependence on the excitation energy, it does not carry any nuclear structure information. The cross section for the DGDR is obtained from Eq. (0.1) by integration over impact parameters, starting from a minimal value $b_{\min }$ to infinity. This minimal value is chosen according to Ref. [5].

In microscopic approaches the strength of the GDR is split among several one-phonon $1_{\alpha}^{-}$states (due to the Landau damping). The wave function $\left|1_{\alpha}^{-}\right\rangle$couples to complex configurations $\mid 1_{\beta}^{-}>$yielding the GDR width. We use the index $\alpha$ for simple configurations and the index $\beta$ for complex ones, respectively. Thus, the wave function of the $i^{t h} 1^{-}$state in the GDR energy region has the form:

$$
\left|1_{i}^{-}>=\sum_{\alpha} S_{i}^{\mathrm{GDR}}(\alpha)\right| 1_{\alpha}^{-}>+\sum_{\beta} C_{i}^{\mathrm{GDR}}(\beta) \mid 1_{\beta}^{-}>
$$

where coefficients $S_{i}^{\mathrm{GDR}}(\alpha)$ and $C_{i}^{\mathrm{GDR}}(\beta)$ can be obtained by diagonalizing the nuclear model Hamiltonian on the set of wave functions (0.2).

The total $E 1$-strength of the GDR excitation from the ground state, $B_{\mathrm{GDR}}(E 1)=\sum_{i}\left|<1_{i}^{-}\right||E 1|\left|0_{\text {g.s. }}^{+}>\right|^{2}$, remains practically the same as in the one-phonon RPA calculation because the direct excitation of complex configurations from the ground state is a few order of magnitude weaker as compared to excitation of one-phonon states. However these complex configurations play a fundamental role for the width of the GDR.

The wave function of the $2^{+}$component of the DGDR states can be written in the similar fashion:

$$
\begin{aligned}
\mid 2_{f}^{+}>= & \sum_{\tilde{\alpha}=\left\{\alpha_{1} \times \alpha_{2}\right\}} S_{f}^{\mathrm{DGDR}}(\tilde{\alpha}) \mid\left[1_{\alpha_{1}}^{-} \times 1_{\alpha_{2}}^{-}\right]_{2^{+}}> \\
& +\sum_{\alpha^{\prime \prime}} \tilde{S}_{f}^{\mathrm{DGDR}}\left(\alpha^{\prime \prime}\right)\left|2_{\alpha^{\prime \prime}}^{+}>+\sum_{\beta^{\prime}} C_{f}^{\mathrm{DGDR}}\left(\beta^{\prime}\right)\right| 2_{\beta^{\prime}}^{+}>
\end{aligned}
$$


In this equation we separated in the first term the doorway $\left[1^{-} \times 1^{-}\right]$DGDR configurations from other twophonon configurations (second term) and complex configurations (the last term). The same equation as (0.3) is valid for the $0^{+}$DGDR states (see Ref. [6] for a discussion on the role of the $0^{+}, 1^{+}$, and $2^{+}$DGDR states in the excitation process).

The total E1-transition strength between the GDR and DGDR, $\sum_{f} \sum_{i}\left|<2^{+}\left(0^{+}\right)_{f}\|E 1\| 1_{i}^{-}>\right|^{2}$, is much larger as compared to that for the GDR excitation, $\sum_{i}\left|<1_{i}^{-}\|E 1\| 0_{\text {g.s. }}^{+}>\right|^{2}$, from the ground state. This is because the former includes transitions not only between doorway GDR and DGDR states but also between complex configurations as well. The enhancement factor should be the ratio between the density of doorway and complex configuration in the GDR energy region. But in the two-step excitation process the sum over intermediate GDR states in Eq. (0.1) reduces the total transition strength for g.s. $\rightarrow G D R \rightarrow D G D R$ to $\sim 2 \cdot\left|B_{\mathrm{GDR}}(E 1)\right|^{2}$ (the factor 2 appears due to the bosonic character of the two phonons which also holds if Landau damping is taken into account). To prove this we substitute the wave functions of the GDR and DGDR states given by Eqs. (0.2,0.3) in expression (0.1). We obtain two terms. The first one corresponds to transitions between doorway GDR and DGDR states (after the GDR is excited from the ground state through its doorway component):

$$
\begin{aligned}
& A_{\mu \mu^{\prime}}=\sum_{i} \sum_{\alpha \alpha^{\prime} \tilde{\alpha}} S_{i}^{\mathrm{GDR}}(\alpha) f_{E 1(\mu)}\left(E_{i}, b\right)<1_{\alpha}^{-}\|E 1\| 0_{g . s .}^{+}> \\
& \quad \times \quad S_{i}^{\mathrm{GDR}}\left(\alpha^{\prime}\right) S_{f}^{\mathrm{DGDR}}(\tilde{\alpha}) f_{E 1\left(\mu^{\prime}\right)}\left(E_{f}-E_{i}, b\right) \\
& \quad \times<\left[1_{\alpha_{1}}^{-} \times 1_{\alpha_{2}}^{-}\right]_{f}\|E 1\| 1_{\alpha^{\prime}}^{-}>\delta_{\alpha_{2}, \alpha^{\prime}}
\end{aligned}
$$

and the second one accounts transitions between complex configurations in the wave functions of Eqs. (0.2,0.3):

$$
\begin{aligned}
B_{\mu \mu^{\prime}}= & \sum_{i} \sum_{\alpha \alpha^{\prime} \beta \beta^{\prime}} S_{i}^{\mathrm{GDR}}(\alpha) f_{E 1(\mu)}\left(E_{i}, b\right)<1_{\alpha}^{-}\|E 1\| 0_{g . s .}^{+}> \\
& \times C_{i}^{\mathrm{GDR}}(\beta) C_{f}^{\mathrm{DGDR}}\left(\beta^{\prime}\right) f_{E 1\left(\mu^{\prime}\right)}\left(E_{f}-E_{i}, b\right) \\
\times< & {\left[1_{\alpha^{\prime}}^{-} \times 1_{\beta}^{-}\right]_{f}\|E 1\| 1_{\beta}^{-}>\delta_{\beta^{\prime},\left[\alpha^{\prime} \times \beta\right]} . }
\end{aligned}
$$

The second reduced matrix element in the above equations is proportional to the reduced matrix element between the ground state and the doorway one-phonon configuration [6].

For a given impact parameter $b$, the function $f_{E 1(\mu)}(E, b)$ can be approximated by a constant value $f_{E 1(\mu)}^{0}$ [14] for the relevant values of the excitation energies. Then the energy dependence can be taken out of summations and orthogonality relations between different components of the GDR wave functions can be applied [12]. The orthogonality relations between the wave functions imply that $\sum_{i} S_{i}^{\mathrm{GDR}}(\alpha) C_{i}^{\mathrm{GDR}}(\beta) \equiv 0$. This means that the term $B_{\mu \mu^{\prime}}$ vanishes. The term $A_{\mu \mu^{\prime}}$ summed over projections and all final states yields a transition probability to the DGDR, $P_{D G D R}\left(E_{f}, b\right)$, which is proportional to $2 \cdot\left|B_{\mathrm{GDR}}(E 1)\right|^{2}$ in second order perturbation theory. This argument was the reason for neglecting the term $B_{\mu \mu^{\prime}}$ in previous calculations of DGDR excitation in Refs. [9, 12, 13 where the coupling of doorway GDR and DGDR states to complex configurations was taken into account.

In Fig. 11 we plot the value of $\chi_{E 1}(E)=$ $\left.2 \pi \int d b b \sum_{\mu} \uparrow f_{E 1(\mu)}(E, b)\right|^{2}$ as a function of energy calculated for the ${ }^{208} \mathrm{~Pb}(640 \cdot \mathrm{A} \mathrm{MeV})+{ }^{208} \mathrm{~Pb}$ reaction. This value corresponds to $\sigma_{\mathrm{GDR}}$ if $B_{\mathrm{GDR}}(E 1)=1$. The square in this figure indicates the location of the DGR in ${ }^{208} \mathrm{~Pb}$. This figure demonstrates that the function $\chi_{E 1}(E)$ changes by $60 \%$ in the GDR energy region. The role of this energy dependence for other effects has been considered in Refs. 河8. Taking into account that onephonon $1_{\alpha}^{-}$configurations are fragmented over a few $\mathrm{MeV}$ [13, when a sufficiently large two-phonon basis is included in the wave function given by Eq. (0.2), the role of the $B_{\mu \mu^{\prime}}$ term in the excitation of the DGDR should be studied in more detail.

To accomplish this task we have performed firstly a simplified calculation in which we used the boson type Hamiltonian:

$$
\begin{aligned}
H= & \sum_{\alpha} \omega_{\alpha} Q_{\alpha}^{\dagger} Q_{\alpha}+\sum_{\beta} \widetilde{\omega}_{\beta} \widetilde{Q}_{\beta}^{\dagger} \widetilde{Q}_{\beta} \\
& +\sum_{\alpha, \beta} U_{\beta}^{\alpha}\left(Q_{\alpha}^{\dagger} \widetilde{Q}_{\beta}+\text { h.c. }\right)
\end{aligned}
$$

where $Q_{\alpha}^{\dagger}$ is the phonon creation operator and $\omega_{\alpha}$ is the energy of this one-phonon configuration; $\widetilde{Q}_{\beta}^{\dagger}$ is the operator for creation of a complex configuration with energy $\widetilde{\omega}_{\beta}$ and $U_{\beta}^{\alpha}$ is the matrix element for the interaction between these configurations. We have assumed that the energy difference between two neighboring one-phonon configurations is constant and equals to $\Delta \omega$. An equidistant spacing with the energy $\Delta \widetilde{\omega}$ was assumed for the complex configurations. We also have used a constant value $U$ for the matrix elements of the interaction. The $B_{\mathrm{GDR}}(E 1)$ value was distributed symmetrically over doorway one-phonon configurations. Thus, the free parameters of this model are: $\Delta \omega, \Delta \widetilde{\omega}, U$, the number of onephonon and complex configurations, and the distribution of the $B_{\mathrm{GDR}}(E 1)$ value among the doorway states. The only condition we want to be satisfied is that the energy spectrum for the GDR photoexcitation is the same as the one known from the experiment.

After all parameters are fixed we diagonalize the model Hamiltonian of Eq. (0.6) on the set of wave functions of Eq. (0.2) for the GDR and on the set of Eq. (0.3) for the DGDR. The diagonalization procedure yields the information on eigen energies of the $1_{i}^{-}$GDR states and on the coefficients $S_{i}^{\mathrm{GDR}}(\alpha)$ and $C_{i}^{\mathrm{GDR}}(\beta)$, respectively. One also obtains information on eigen energies of the $2_{f}^{+}$ or $0_{f}^{+}$DGDR states and the coefficients $S_{f}^{\mathrm{DGDR}}(\tilde{\alpha})$ and $C_{f}^{\mathrm{DGDR}}\left(\beta^{\prime}\right)$, respectively. With this information we are able to study the role of the $B_{\mu \mu^{\prime}}$ term in the excitation 
of the DGDR in RHIC.

The big number of free parameters allows an infinite number of suitable choices. In fact, not all of the parameters are really independent. For example, the increase in the number of simple or complex configurations goes together with the decreasing of the value of $U$. This is necessary for a correct description of the GDR photoabsorption cross section. This makes it possible to investigate the role of the $B_{\mu \mu^{\prime}}$ term in different conditions of weak and strong Landau damping and for different density of complex configurations. In our calculations we vary the number of collective doorway states from one to seven and the number of complex configurations from 50 to 500 . The value of $U$ then changes from about 100 to $500 \mathrm{keV}$. The results of one of these calculations for the excitation of the $2^{+}$component of the DGDR in ${ }^{208} \mathrm{~Pb}(640 \cdot \mathrm{A} \mathrm{MeV})+{ }^{208} \mathrm{~Pb}$ collisions are presented in Fig. 2. For a better visual appearance the results are averaged with a smearing parameter equal to $1 \mathrm{MeV}$. The dashed curve shows the results of a calculation in which $\sigma_{\mathrm{DGDR}}^{A}(E) \equiv \sigma_{\mathrm{DGDR}}(E) \sim \int d b b\left|A_{\mu \mu^{\prime}}\right|^{2}$ and the results of another one in which $\sigma_{\mathrm{DGDR}}^{A+B}(E) \equiv \sigma_{\mathrm{DGDR}}(E) \sim$ $\int d b b\left|A_{\mu \mu^{\prime}}+B_{\mu \mu^{\prime}}\right|^{2}$ are represented by a solid curve.

Our calculation within this simple model indicates that the role of the $B_{\mu \mu^{\prime}}$ term in the second order perturbation theory is negligibly small, although the total $B(E 1)$ strength for transitions between complex GDR and DGDR configurations, considered separately, is more than two orders of magnitude larger than the ones between doorway GDR and DGDR configurations. The value $\Delta \sigma=\left(\sigma_{\mathrm{DGDR}}^{A+B}-\sigma_{\mathrm{DGDR}}^{A}\right) / \sigma_{\mathrm{DGDR}}^{A}$, where $\sigma_{\mathrm{DGDR}}^{A(A+B)}=$ $\int \sigma_{\mathrm{DGDR}}^{A(A+B)}(E) d E$, changes in these calculations from $1 \%$ to $2.5 \%$. The results practically do not depend on the number of complex configurations accounted for. The maximum value of $\Delta \sigma$ is achieved in a calculation with a single doorway GDR state (no Landau damping). This is because the value of $U$ is the larger in this case and the fragmentation of the doorway state is stronger. Thus, in such a situation, the energy dependence of the reaction amplitude modifies appreciably the orthogonality relations. But in general the effect is marginal.

We also performed a calculation with more realistic wave functions for the GDR and DGDR states taken from our previous studies [9] which were based on microscopic QPM (Quasiparticle Phonon Model) [16]. These wave functions include 6 and 21 doorway states for the GDR and DGDR, respectively. The complex configurations are two-phonon states for the GDR and three-phonon states for the DGDR. The energies of the doorway states and complex configurations were obtained from RPA equations and the matrix elements of the interaction, $U_{\beta}^{\alpha}$, were calculated on a microscopic footing without free parameters by making use of the QPM Hamiltonian and internal fermion structure of phonons. The value $\Delta \sigma$ equals in this realistic calculation to $0.5 \%$. This result is not surprising because realistic calculations with only twophonon complex configurations, and a limited number of them, somewhat underestimate the GDR width which is crucial for the modification of the orthogonality relations.

In conclusion, we investigated the role of transitions between complex GDR and DGDR configurations within second-order perturbation theory for the DGDR excitation in RHIC. We have proved that these transitions play a marginal role in the process under consideration and it is sufficient to take into account only transitions between the ground state and doorway GDR and DGDR configurations.

V.Yu. P. thanks for the hospitality at the Instituto de Física of the Universidade Federal do Rio de Janeiro, where this research has been performed, the $\mathrm{CNPq}$ for the financial support and Prof. P.F. Bortignon for fruitful discussions. This work was partially supported by the RFBR (grant 96-15-96729), by the Heisenberg-Landau program, by the FUJB/UFRJ and by the $\mathrm{MCT} / \mathrm{FINEP} / \mathrm{CNPq}(\mathrm{PRONEX})$ (contract 41.96.0886.00)

[1] J.L. Ritman et al. (TAPS collaboration), Phys. Rev. Lett. 70, 533 and 2659 (1993).

[2] R. Schmidt et al. (LAND collaboration), Phys. Rev. Lett. 70, 1767 (1993).

[3] C.A. Bertulani, and V.G. Zelevinsky, Phys. Rev. Lett. 71, 967 (1993); Nucl. Phys. A 568931 (1994).

[4] V.Yu. Ponomarev, E. Vigezzi, P.F. Bortignon, R.A. Broglia, G. Colò, G. Lazzari, V.V. Voronov, and G. Baur, Phys. Rev. Lett. 72, 1168 (1994).

[5] C.A. Bertulani, L.F. Canto, M.S. Hussein, and A.F.R. de Toledo Piza, Phys. Rev. C 53, 334 (1996).

[6] C.A. Bertulani, V.Yu. Ponomarev, and V.V. Voronov, Phys. Lett. B 388, 457 (1996).

[7] E.G. Lanza, M.V. Andrés, F. Catara, Ph. Chomaz, and C. Volpe, Nucl. Phys. A 613, 445 (1997).

[8] P.F. Bortignon, and C.H. Dasso, Phys. Rev. C 56, 574 (1997).

[9] V.Yu. Ponomarev, and C.A. Bertulani, Phys. Rev. Lett. 79, 3853 (1997).

[10] K. Boretzky et al. (LAND collaboration), Phys. Lett. B 384, 30 (1996).

[11] V.G. Soloviev, A. Arima, and N.D. Dang, Phys. Rev. C 56, R603 (1997).

[12] V.Yu. Ponomarev, P.F. Bortignon, R.A. Broglia, E. Vigezzi, and V.V. Voronov, Nucl. Phys. A 599, 341c (1996).

[13] V.Yu. Ponomarev, P.F. Bortignon, R.A. Broglia, and V.V. Voronov, Z. Phys. A 356, 251 (1996).

[14] C.A. Bertulani, and G. Baur, Phys. Rep. 163, 299 (1988).

[15] A. Winther and K. Alder, Nucl. Phys. A 319, 518 (1979).

[16] V.G. Soloviev, Theory of Atomic Nuclei: Quasiparticles and Phonons (Institute of Physics, Bristol, 1992). 
FIG. 1. The energy dependence of the ${ }^{208} \mathrm{~Pb}(640 \cdot \mathrm{A} \mathrm{MeV})+{ }^{208} \mathrm{~Pb}$ reaction function calculated within first order perturbation theory. The square indicates the location of the GDR in ${ }^{208} \mathrm{~Pb}$.

FIG. 2. The cross section for the excitation of the $2^{+}$component of the DGDR in the reaction ${ }^{208} \mathrm{~Pb}$ $(640 \cdot \mathrm{A} \mathrm{MeV})+{ }^{208} \mathrm{~Pb}$, calculated within second order perturbation theory. The dashed curve shows the contribution of the E1-transition between doorway GDR and DGDR configurations only. The solid curve is a sum of the above result and the contribution of the E1-transitions between complex GDR and DGDR configurations. See text for details. 


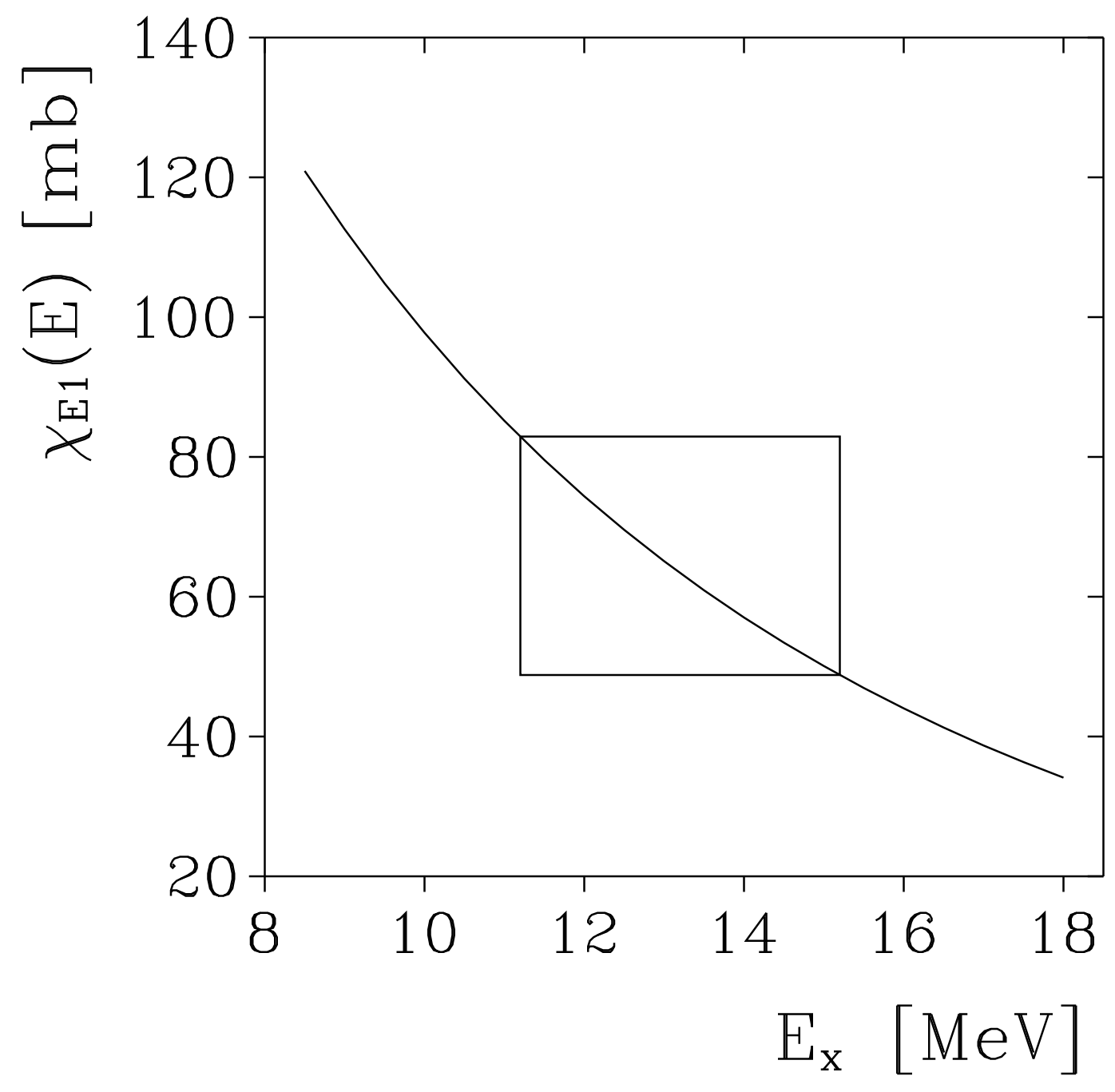




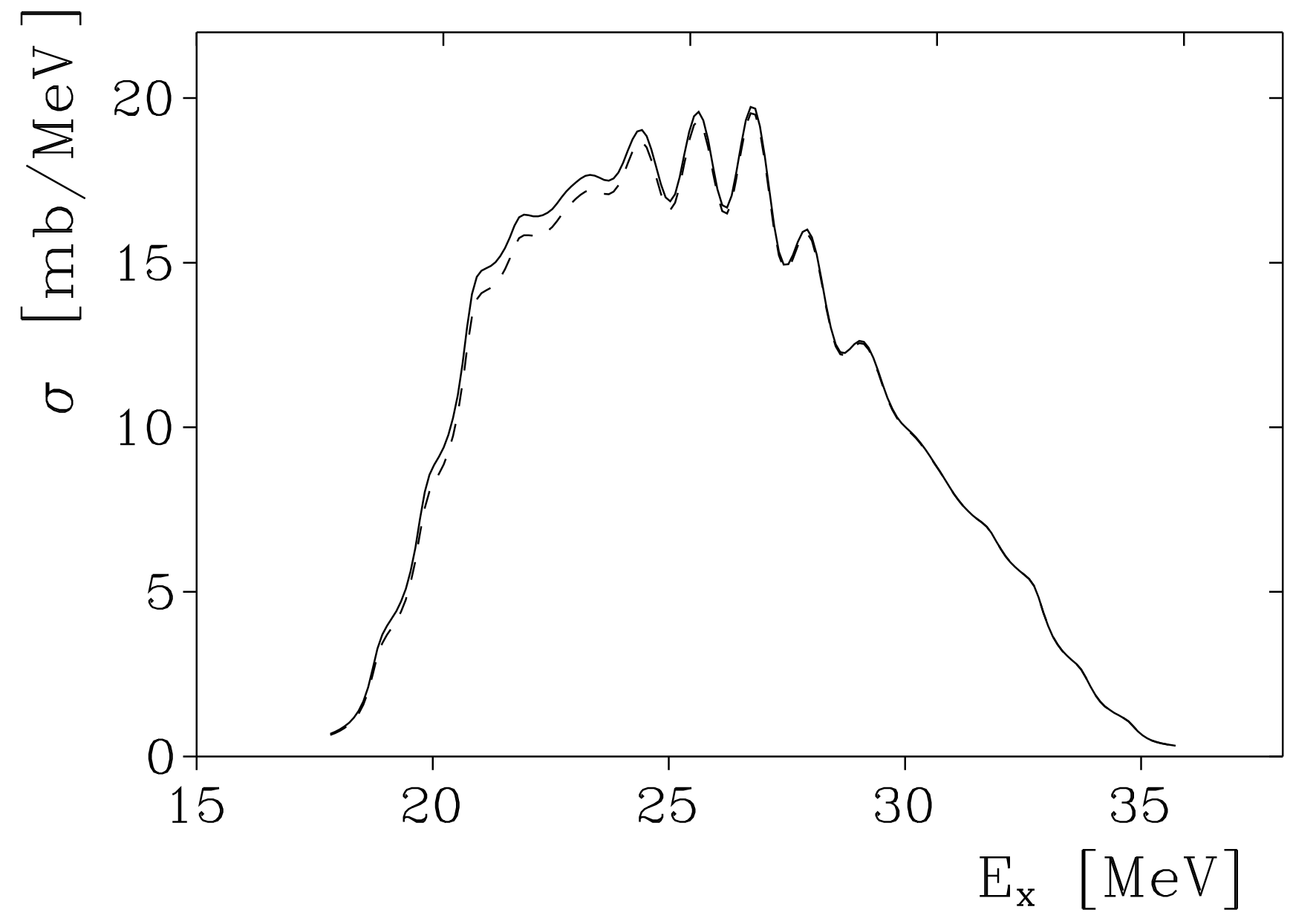

